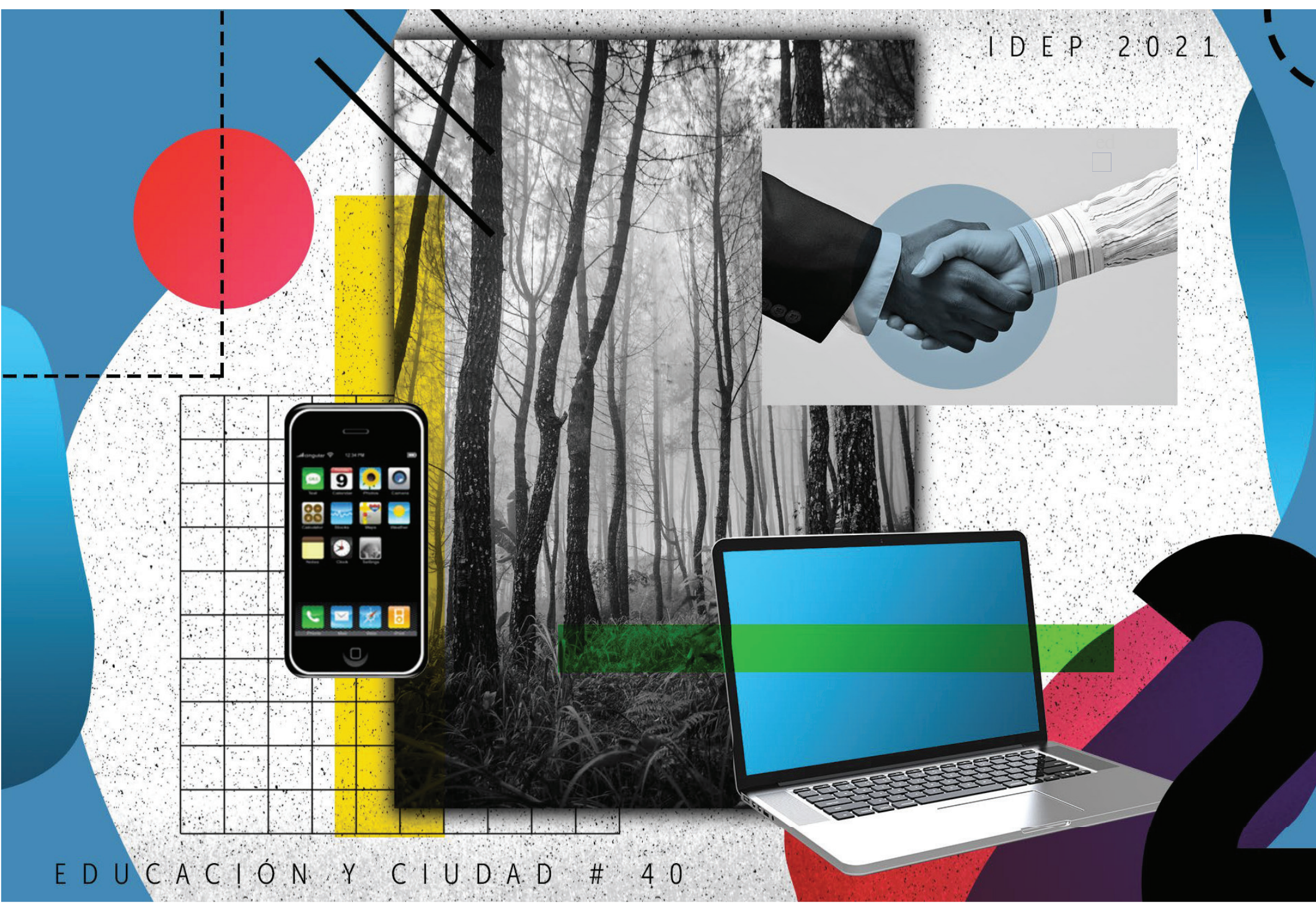

\title{
Análisis de problemas socioambientales: una experiencia desde lo virtual
}

ANALYSIS OF SOCIO-ENVIRONMENTAL PROBLEMS: AN EXPERIENCE FROM THE VIRTUAL ANÁLISE DE PROBLEMAS SOCIOAMBIENTAIS: UMA EXPERIÊNCIA DO VIRTUAL 


\section{Laura Yesenia Solano ${ }^{1}$}

1. Magíster en Geografía, Universidad de los Andes; Licenciada en Ciencias Sociales, Universidad Pedagógica Nacional; Docente de ciencias sociales para grados $4^{\circ}$ y $5^{\circ}$, Liceo de Colombia Bilingüe; ORCID: https://orcid.org/0000-0002-9647-0597; correo electrónico: laurasolanov@gmail.com

Citar artículo como:

Solano, L. (2021, Enero-Junio). Análisis de problemas socioambientales: una experiencia desde lo virtual. Revista Educación y Ciudad, No. 40, pp. 35-48. //doi.org/10.36737/01230425.n40.2021.2455

DOI: https://doi.org/10.36737/01230425.n40.2021.2455

Fecha de recepción: 3 de agosto de 2020 / Fecha de aprobación: 4 de noviembre de 2020

\section{Resumen}

El presente artículo expone una experiencia de aula virtual sobre la elaboración de un Podcast ambiental. La actividad tuvo como objetivo aprender sobre la geografía nacional a través de la exploración de los conflictos socioambientales que le han aquejado. Bajo los lineamientos de la Enseñanza para la Comprensión, se implementó una unidad didáctica que desarrolló habilidades en los estudiantes según los estándares de competencias en ciencias sociales. El documento concluye que la propuesta es una alternativa atractiva y eficiente de enseñanza virtual para la comprensión de conflictos de este tipo.

Palabras clave: Conflictos socioambientales, parques naturales nacionales, aula virtual, enseñanza para la comprensión.

\begin{abstract}
This article presents a virtual classroom experience on making an environmental podcast. This activity aimed to learn about national geography through the exploration of the socio-environmental conflicts that had arisen there. Under the guidelines of Teaching for Understanding, this didactic unit increased students' skills according to the development of social science competencies. The document concludes that this proposal is an attractive and efficient virtual teaching alternative for understanding conflicts of this type.
\end{abstract}

Keywords: Socio-environmental conflicts, national natural parks, virtual classroom, teaching for understanding.

\section{Resumo}

Este artigo apresenta uma experiência em sala de aula virtual sobre como criar um podcast ambiental. Esta atividade teve como objetivo conhecer a geografia nacional através da exploração dos conflitos socioambientais que aí surgiram. Sob as diretrizes de Ensino para a Compreensão, foi implementada uma unidade didática que desenvolvia habilidades nos alunos de acordo com os padrões de competência em ciências sociais. O documento conclui que esta proposta é uma alternativa de ensino virtual atraente e eficiente para a compreensão de conflitos desse tipo.

Palavras-chave: Conflitos socioambientais, parques naturais nacionais, sala de aula virtual, ensino para a compreensão. 


\section{Introducción}

L a aparición del COVID-19 en el escenario mun$\perp$ dial creó una serie de paradigmas inexplorados que impactaron seriamente todas las esferas de la sociedad, entre ellas, el escenario educativo. En este contexto se inicia, de forma casi inmediata, la implementación de aulas completamente virtuales en todas aquellas instituciones que podían permitirse comprar plataformas y garantizar su acceso para toda la comunidad educativa.

Dadas estas particularidades, inicia una carrera contra el tiempo para establecer ajustes a la estructura curricular y el reordenamiento de los tiempos, lenguajes y medios de aula (Norman y Daza, 2020); lo cual implicó, por tanto, reflexionar acerca de los esquemas transitorios que este reto significa en cuanto a evaluación, métodos, objetos, etc. Entendida como modalidad o metodología, la educación virtual en tiempos de pandemia permitió explorar y afrontar un sinfín de posibilidades y retos didácticos, metodológicos e incluso psicosociales. La urgencia para adaptarlos era urgente.

A pesar de la incertidumbre, tal desafío coyuntural permitió a muchos docentes acelerar el proceso de creación e implementación de estrategias pedagógicas sobre la marcha $\mathrm{y}$, justamente, un ejemplo exitoso de este ejercicio creativo han sido las estrategias que se tuvo la oportunidad de implementar en la enseñanza de la geografía de Colombia, para grado quinto de primaria, en una institución internacional de básica y media privada al norte de la ciudad de Bogotá. Con el tema se buscó reconocer la estructura social, política y económica de algunos problemas socioambientales tejidos en el territorio nacional, vinculados a la diversidad de intereses y percepciones acerca del uso de su naturaleza.

Un Podcast ambiental. La actividad tuvo como objetivo construir y evaluar de forma integral el desarrollo de competencias en la asignatura, entre ellas, el pensamiento social, la interpretación y análisis de perspectivas y el pensamiento reflexivo y sistémico.

El presente artículo busca, entonces, describir la experiencia pedagógica que surgió de tal necesidad didáctica, vinculada a la búsqueda de nuevos métodos para la virtualidad. El escrito se dividirá esencialmente en tres partes: la primera, explica la importancia del análisis de los conflictos ambientales para la formación crítica de los estudiantes: la segunda, expone en detalle el paso a paso de la unidad didáctica, poniendo sobre la mesa los objetivos, herramientas y métodos implementados para preparar, ejecutar y evaluar el proyecto de aula; finalmente, en la tercera parte, se enumeran algunos resultados y conclusiones de la experiencia.

\section{Analizar conflictos socioambientales en el aula}

La geografía escolar tradicional ha dejado de lado durante mucho tiempo el carácter crítico de la disciplina (Cely y Moreno, 2008). Es común encontrar en las mallas curriculares institucionales unlargo compendio de temasquealudenala memoria y suprimen el análisis crítico de los fenómenos espaciales (Rodríguez, 2000). Autores como Souto (1997) apuestan por identificar la geografía como una disciplina social e históricamente dinámica y 
cambiante, que busca analizar la complejidad del espacio e intenta examinar sus poderes, jerarquías, movimientos y desacuerdos. Los conflictos socio ambientales son, entonces, uno de los tantos ámbitos de interés de la disciplina.

Dichos problemas, para autores como Martínez (2008) se refieren a trastoques entre los intereses y valoraciones de un entorno natural, que entran en disputa por el acceso, uso, distribución y calidad de los recursos naturales disponibles. Generalmente, las confrontaciones se presentan entre la institucionalidad estatal y una comunidad habitante, sin embargo, también pueden darse choques entre sujetos externos y la comunidad o dentro de la misma comunidad habitante.

Autores como Souto (1997), y Jiménez y Gaite (1996), han escrito ampliamente acerca de la importancia pedagógica de analizar los problemas socioambientales dentro del aula formal, para el desarrollo de habilidades inherentes a las ciencias sociales. De igual forma, coinciden en comprender el ejercicio como una herramienta esencial para la formación de ciudadanos del siglo XXI: conscientes de su entorno, críticamente activos y orientados a la resolución de problemas comunes.

Partiendo de ello, la unidad didáctica del trimestre escolar estuvo orientada a reconocer las particularidades de la geografía nacional colombiana, a través de la exploración de los conflictos socioambientales que se han presentado por su uso o control. Buscaba, en esencia, que los estudiantes fueran capaces de analizar una problemática, de contrastar las diferentes posturas y, finalmente, de elaborar propuestas de solución que tomaran en cuenta todas las dimensiones posibles.
A fin de responder a dicha complejidad didáctica, se escogieron algunos Parques Nacionales Naturales de Colombia (PNN) como unidades territoriales con claros conflictos vinculados al uso de la naturaleza. Por un lado, parques como el PNN Cocuy, PNN Sumapaz y el Santuario de Fauna y Flora (SFF) Corales del Rosario, presentan agudos debates sobre la pertinencia ambiental, social y económica del turismo. En todos los casos la actividad turística ha impactado directamente a las comunidades locales (campesinos e indígenas) y al medio que habitan, generando un choque de intereses entre promotores, turistas y habitantes.

Por otro lado, se expuso la situación del PNN Tinigua, donde la deforestación por ganadería y cultivos ilícitos en plena selva amazónica (tras el acuerdo de paz con las Fuerzas Armadas Revolucionarias de Colombia -FARC) pone en riesgo la estabilidad biológica de un territorio ancestralmente indígena; problemática que ha generado un enfrentamiento entre entidades ambientales, disidencias de las FARC, campesinos e indígenas. Mientras que, por su parte, en el PNN Chingaza se debate el futuro del abastecimiento hídrico de más de 9 millones de personas de Bogotá, que se encuentran bajo la constante amenaza de un daño ambiental irreparable; campesinos, organizaciones ambientales, la empresa de acueducto y los propios habitantes de la ciudad comparten, traslapan y enfrentan intereses diversos sobre su uso.

\section{Proyecto de aula: Un podcast ambiental}

Partiendo de lo expresado por el Ministerio de Educación Nacional de Colombia, la educación virtual 
(o educación en línea) se refiere a los programas de formación emplazados en el escenario virtual con la ayuda de las tecnologías para la información y la Comunicación (TIC), para establecer nuevas formas de enseñar y aprender (MEN, 2020). La virtualidad ofrece, en escenarios óptimos, alternativas frente a aspectos como los ritmos, formatos, contenidos y fuentes de información; de igual manera, brinda múltiples opciones para el diseño y utilización de materiales que generalmente no son, o no pueden ser, involucrados en el aula presencial.

En este sentido, y a pesar de la contingencia mundial, las TIC y el internet hicieron posible dinamizar la educación virtual para estudiantes y docentes que muy probablemente no habían estado expuestos a ella. Dicha naturaleza del ciberespacio permitió emplear con mayor facilidad recursos como videos, animaciones y videojuegos con enfoque educativo (Cáceres, 2020). Sin dejar de lado las directrices pedagógicas que orientan el quehacer del aula presencial, esta experiencia retomó algunas ventajas de la virtualidad, utilizándolas para conectar los problemas socioambientales del territorio colombiano con la apropiación de nuevos modelos de enseñanza-aprendizaje a través de lo "virtual".

En primera instancia, la directriz pedagógica que orientó la experiencia fue la Enseñanza para la Comprensión (EPC) que, según Stone (1999), se trata de un marco metodológico de enseñanza orientado al desarrollo de procesos cognitivos superiores, como pensar de forma crítica, trabajar de forma colaborativa para encontrar soluciones y propiciar la creatividad. En esta propuesta, la evaluación busca fomentar el uso del conocimiento como pilar de solución para los problemas que sean planteados, pues así se garantiza una comprensión real de las temáticas y habilidades propuestas.

En la misma línea propuesta por Stone, esta implementación metodológica compromete la consecución de una serie de pasos claros para el estudiante y el educando: en primer lugar, crear un tópico generativo o hilo conductor que relacione temas y problemáticas de interés para los estudiantes (Stone, 1999): luego, unas metas de comprensión específicas de conocimiento público, como norte de cada unidad; y, a continuación, estructurar una serie limitada y concreta de desempeños de comprensión, en los que se organizan las actividades secuenciadas de los estudiantes. Siguiendo esta propuesta, el desarrollo de nuestra unidad se dividió en tres partes: exploración, investigación guiada y proyecto final de síntesis.

Para la primera fase de exploración (Tabla 1), los estudiantes empezaron a analizar una problemática socioambiental interesante; caracterizaron su bioma, lo localizaron y reconocieron su fragilidad. Luego de ello, identificaron la diversidad de actores relacionados con la problemática para, posteriormente, reconocer que esos mismos actores se mueven desde una serie de intereses dispares que muchas veces se superponen o chocan; finalmente, evaluaron y diseñaron soluciones acordes para aquellas necesidades disímiles. Como puede verse, esta primera fase cuenta con una compañía constante de la docente, quien brinda pautas concretas para el desarrollo de una indagación. 
Tabla 1. Exploración e investigación guiada. Grado quinto, 2020

\begin{tabular}{|c|c|}
\hline \multicolumn{2}{|r|}{ Unidad didáctica: problemas socio ambientales en Colombia } \\
\hline Tópico generativo & Meta de comprensión \\
\hline $\begin{array}{c}\text { ¿Están en peligro } \\
\text { nuestros parques } \\
\text { nacionales? }\end{array}$ & $\begin{array}{l}\text { El estudiante desarrollará sus destrezas para comprender el territorio colombiano, } \\
\text { identificando las diferentes perspectivas que giran alrededor de las problemáticas am- } \\
\text { bientales, a través de la creación de un Podcast que trata un problema de este tipo }\end{array}$ \\
\hline \multicolumn{2}{|r|}{ Desempeños de comprensión } \\
\hline \multirow{6}{*}{$\begin{array}{l}\text { Exploración } \\
\text { (3 sesiones) }\end{array}$} & $\begin{array}{l}\text { Paso 1: Visualización de una noticia sobre una problemática ambiental actual (Cierre } \\
\text { del PNN El Cocuy por exceso de turismo) }\end{array}$ \\
\hline & $\begin{array}{l}\text { Paso 2: Búsqueda de información por internet respecto a las condiciones geográficas } \\
\text { del páramo y súper páramo. Actividad 1: Formular un encabezado de noticia en el que } \\
\text { se relacionen las condiciones ambientales con el problema tratado }\end{array}$ \\
\hline & $\begin{array}{l}\text { Paso 3: Identificación del problema. Actividad 2: Formular un primer párrafo de la } \\
\text { noticia exponiendo en dos frases el problema tratado en la misma }\end{array}$ \\
\hline & $\begin{array}{l}\text { Paso 4: Reconocimiento de actores y argumentos dentro del problema subrayando con } \\
\text { claves de colores. Actividad 3: Redactar el segundo párrafo de la noticia exponiendo } \\
\text { posiciones y argumentos }\end{array}$ \\
\hline & $\begin{array}{l}\text { Paso 5: Encontrar las acciones concretas tomadas por los actores. Actividad 4: Escribir } \\
\text { un párrafo de conclusión formulando una posible solución al problema planteado en la } \\
\text { noticia }\end{array}$ \\
\hline & $\begin{array}{l}\text { Paso 6: Evaluación de análisis y alternativas. Actividad 5: Las columnas son publicadas } \\
\text { en la plataforma de Nearpod y son retroalimentadas por la comunidad }\end{array}$ \\
\hline \multirow[t]{8}{*}{ Investigación guiada } & Paso 1: Selección de uno de los problemas socio ambientales propuestos: \\
\hline & - Turismo descontrolado en el PNN Sumapaz \\
\hline & - Construcciones ilegales en PNN Corales del Rosario \\
\hline & - Deforestación por ganadería en PNN Tinigua \\
\hline & - Extracción de agua para Bogotá del PNN Chingaza \\
\hline & $\begin{array}{l}\text { Paso 2: Lectura de una noticia y visualización de un documental sobre las condiciones } \\
\text { biológicas y geográficas del parque nacional seleccionado }\end{array}$ \\
\hline & $\begin{array}{l}\text { Paso 3: Se realizan individualmente los pasos } 3 \text { al } 5 \text { de la fase exploratoria, con sus } \\
\text { respectivas actividades }\end{array}$ \\
\hline & $\begin{array}{l}\text { Paso 4: Los estudiantes comparten los textos en forma oral, utilizando la herramienta } \\
\text { grupo de Teams; también se cuenta con retroalimentación colectiva (NOTA DE CALI- } \\
\text { FICACIÓN \#1) }\end{array}$ \\
\hline
\end{tabular}


educación

Ciudad

Ya en la fase de investigación guiada se entregaron las herramientas requeridas por el estudiante para iniciar un primer trabajo en solitario; vale la pena aclarar que también se le brindó la posibilidad de seleccionar un caso que le fuera atractivo o cercano, con el cual pudiera comprometerse; junto a ello, además de dar una noticia vinculada a la problemática, se envió un documental relacionado, para que así le fuese posible reconocer más claramente las características del bioma tratado y las razones por las que es frágil frente a la situación problema.
El proyecto final, que sintetizaría todo lo aprendido, consistió en la realización de un Podcast ambiental (Tabla 2). Como preparación para la grabación, se realizó un guión de conversación que debía seguir una serie de pasos (actividad evaluada y retroalimentada por la docente como nota); a partir de dicha secuencia se desarrollaría la exposición del problema, sus actores, perspectivas y posibles soluciones. La actividad fue grabada durante la clase utilizando una página de internet que permite el envío de un enlace público de reproducción (sistema que hace mucho más sencilla la entrega y co-evaluación del proyecto).

Tabla 2. Proyecto final de síntesis (PFS). Grado quinto, 2020

\begin{tabular}{|c|c|}
\hline Fase & Actividades \\
\hline $\begin{array}{l}\text { Proyecto final de } \\
\text { síntesis }\end{array}$ & $\begin{array}{l}\text { Paso 1: Escucha un Podcast de radio que trate sobre el derretimiento de los nevados en } \\
\text { Colombia. Actividad 1: Los estudiantes hacen una lista de las fases de presentación del } \\
\text { tema, que incluye: }\end{array}$ \\
\hline & $\begin{array}{l}\text { - Saludo y presentación del locutor } \\
\text { - Presentación del problema y actores (¿Cuál es el problema y por qué lo es?; ¿quién } \\
\text { lo está ocasionando?; ¿qué y quiénes están siendo afectados?) } \\
\text { - Presentación de datos y cifras importantes relacionadas con el problema (¿Qué } \\
\text { ecosistema está siendo alterado?; ¿por qué es frágil?; ¿por qué es tan importante } \\
\text { este PNN?) } \\
\text { - Exponer las fuentes utilizadas para sustentar la información (periódicos, fechas, } \\
\text { nombre del documental) } \\
\text { - Creación de entrevistas que retomen y expongan las posturas de los actores } \\
\text { involucrados en el problema (¿Qué piensan?; ¿por qué?; ¿¿uál es su motivación o } \\
\text { interés?) } \\
\text { - Análisis de las entrevistas (¿Existe un choque de intereses?: ¿por qué no se ha } \\
\text { encontrado una solución?) }\end{array}$ \\
\hline
\end{tabular}




\begin{tabular}{|c|c|}
\hline Fase & \multicolumn{1}{c|}{ Actividades } \\
\hline $\begin{array}{c}\text { Proyecto final de } \\
\text { síntesis }\end{array}$ & $\begin{array}{l}\text { Exposición de conclusiones sobre el problema y las posturas involucradas. Ellas } \\
\text { deben generar una posible solución, teniendo en cuenta todas las posturas del } \\
\text { escenario }\end{array}$ \\
& $\begin{array}{l}\text { Cierre y despedida. } \\
\text { Paso 2: Desde el programa de actividades, los estudiantes seleccionan otro problema de } \\
\text { la lista para realizar su propio Podcast. Actividad 2: Escriben el guión en un cuaderno } \\
\text { virtual; se verifican avances y se da retroalimentación en tiempo real (NOTA DE CALI- } \\
\text { FICACIÓN \#2) } \\
\text { Paso 3: Práctica del diálogo con otros compañeros a través de herramienta grupal. Acti- } \\
\text { vidad 3: Primera retroalimentación entre pares del PFS utilizando la rúbrica }\end{array}$ \\
$\begin{array}{l}\text { Paso 4: Actividad 4: Grabación del Podcast en la página Vocarroo.com Se sugiere utili- } \\
\text { zar efectos de sonido; el enlace de dicho trabajo se sube a la red en un cuaderno virtual } \\
\text { grupal }\end{array}$ \\
$\begin{array}{l}\text { Paso 5: Retroalimentación final. Actividad 5: Se seleccionan 4 trabajos y se les retroali- } \\
\text { menta tomando en cuenta los criterios observados; así mismo, se generan comentarios } \\
\text { (NOTA DE CALIFICACIÓN \#3) }\end{array}$ \\
\hline
\end{tabular}

El desarrollo paso a paso del Podcast tuvo como objetivo el crecimiento de competencias relativas a las ciencias sociales, a través del análisis de una problemática socio ambiental, que involucró: pensamiento social, interpretación y análisis de perspectivas, y pensamiento reflexivo y sistémico. Esta forma de evaluar se ve claramente definida en la rúbrica de evaluación mediante la cual los estudiantes fueron retroalimentados (Tabla 3), que incluyó distintos criterios y su respectiva intención pedagógica, de la siguiente forma. 
Tabla 3. Rúbrica de evaluación para el Podcast ambiental. Grado quinto, 2020

\begin{tabular}{|c|c|c|}
\hline Competencia & Criterios de evaluación & $\begin{array}{l}\text { Desempeño superior/ } \\
\text { /alto/bajo }\end{array}$ \\
\hline \multirow{3}{*}{$\begin{array}{l}\text { Pensamiento } \\
\quad \text { social }\end{array}$} & Ubica espacialmente la problemática & \\
\hline & $\begin{array}{l}\text { Reconoce la relación entre el problema y sus características } \\
\text { geográficas particulares (bioma, relieve, hidrografía, } \\
\text { fragilidad) }\end{array}$ & \\
\hline & Aplica mecanismos de participación dentro de la solución & \\
\hline \multirow{5}{*}{$\begin{array}{l}\text { Interpretación } \\
\text { y análisis de } \\
\text { perspectivas }\end{array}$} & $\begin{array}{l}\text { Identifica a cada uno de los actores dentro de la } \\
\text { problemática }\end{array}$ & \\
\hline & Reconoce a los responsables del fenómeno & \\
\hline & $\begin{array}{l}\text { Analiza y contrasta, en al menos tres entrevistas, las } \\
\text { perspectivas, intereses, vulnerabilidades y prioridades de } \\
\text { los actores frente al problema }\end{array}$ & \\
\hline & $\begin{array}{l}\text { Menciona por lo menos dos cifras relevantes sobre el } \\
\text { problema para explicar su gravedad o impacto }\end{array}$ & \\
\hline & Menciona al menos tres fuentes de información & \\
\hline \multirow{4}{*}{$\begin{array}{c}\text { Pensamiento } \\
\text { reflexivo y } \\
\text { sistémico }\end{array}$} & $\begin{array}{l}\text { Identifica adecuadamente la problemática a nivel social y } \\
\text { ambiental }\end{array}$ & \\
\hline & Reconoce al menos un impacto ambiental y uno social & \\
\hline & Evalúa las soluciones tomadas por los actores & \\
\hline & $\begin{array}{l}\text { Propone una solución que ponga en diálogo todas las } \\
\text { perspectivas del problema }\end{array}$ & \\
\hline
\end{tabular}




\section{Pensamiento social}

De acuerdo con el Ministerio de Educación Nacional, la competencia de pensamiento social evalúa "la capacidad del estudiante para usar conceptos básicos de las ciencias sociales que permiten la comprensión de problemáticas y fenómenos sociales, políticos, económicos culturales y geográficos" (2014, p. 16). En otras palabras, las actividades deben buscar que los estudiantes usen el conocimiento del que disponen (sobre conceptos, épocas, lugares, etc.) para analizar las problemáticas históricas o contemporáneas.

Partiendo de tal definición, el trabajo con el Podcast buscaba evaluar la capacidad de los estudiantes para relacionar las condiciones biológicas del PNN con la problemática; así, por una parte, conectaron la fragilidad del suelo del páramo en Chingaza o Sumapaz con los efectos negativos causados por las constantes pisadas de los turistas; o relacionaron la problemática ambiental del calentamiento global con el deshielo del Cocuy y el pisoteo de los caballos en el súper páramo, tal como se hace evidente a continuación:

Allá en Tinigua está pasando algo muy grave, queridos oyentes. Están quemando la selva para tener ganadería. La selva se daña cuando la queman, los huecos de flora que quedan por esas acciones hacen que los animales se queden sin hábitat (Podcast, No. 4).

Los estudiantes también debían utilizar temáticas ya tratadas, como los mecanismos de participación, para proponer soluciones democráticas a las problemáticas. Algunos de ellos propusieron tutelas, consultas populares, cabildos abiertos e incluso revocatorias del mandato, cuando el problema tenía su origen en licencias otorgadas por la alcaldía local o municipal.

\section{Interpretación y análisis de perspectivas}

Continuando con las competencias trabajadas, la interpretación y análisis de perspectivas se refiere a la capacidad de "reconocer perspectivas, analizarlas, en particular por medio del examen de los argumentos que presente el representante (individuo o colectivo) de determinada perspectiva o posición (en situaciones cotidianas, interpersonales o históricas)" (Ministerio de Educación, 2014, p. 16). Este criterio también compete al uso de evidencias o fuentes, la valoración de prejuicios e intenciones implícitas en los argumentos, y la revisión de posibles choques entre dichas perspectivas.

Para la creación del Podcast esta competencia toma especial relevancia, pues pone en evidencia la naturaleza compleja de los conflictos socioambientales. Los estudiantes recurrieron a estrategias como modular su voz, solicitar ayuda de sus familiares e inclusive a usar voces modificadas por programas digitales, para simular entrevistas que demostraran la diversidad de actores y perspectivas del problema.

En primera instancia, se les solicitó identificar aquellos actores relacionados directamente con el conflicto; campesinos, turistas, empresas turísticas, terratenientes, el Sistema de Parques Nacionales Naturales (SINAP) e indígenas, por nombrar algunos, fueron los reseñados más comúnmente. Luego debían hacer una lista que incluyera los argumentos que daban forma a las perspectivas de dichos 
actores frente al problema ambiental tratado; así, muchos estudiantes localizaron argumentos como:

Yo soy un indígena U'wa y no quiero que me dañen más mi tierra. Aquí nosotros somos los que cuidamos, esa gente de allá viene con caballos y pisan el suelo, eso lo dañan mucho (Podcast, No. 7).

El análisis de perspectivas permitió a los estudiantes reconocer que también existían choques y alianzas entre los diversos intereses de los actores, tal como se evidencia a continuación:

Los señores de parques nacionales están intentando que escuchen a los campesinos para que también los dejen cultivar en esta tierra, pero los ganaderos no quieren que se les quite todo el poder que tienen allá (Podcast, No. 12).

Esto posibilitó, a su vez, comparar y contrastar los distintos intereses para, posteriormente, evaluar el impacto de ciertas decisiones tomadas frente a la problemática. Algunos llegaron a caracterizar económica y políticamente a sus actores, solo para reconocer lo vulnerables o poderosos que eran frente a las decisiones ya tomadas, tal como se demuestra en el siguiente fragmento:

Mire, yo soy un campesino, yo no tengo más que la papa aquí en el páramo. No, no puedo creer que usted me diga que es mejor que hagamos el turismo, y yo sé que eso no me da a mí la plata que usted dice que me va a dar. No me engañe con eso del turismo hecho por las empresas de afuera (Podcast, No. 32).

Por último, esta competencia también permitió a los estudiantes manejar distintas fuentes dentro de su discurso, ya que debían citar al menos dos para dar una referencia clara del origen de su información. Debido a su estadio de desarrollo, por el momento no se solicitó evaluar la veracidad de dichas fuentes.

\section{Pensamiento reflexivo y sistémico}

Por último, la competencia de pensamiento reflexivo y sistémico se entiende como "la habilidad de reconstruir y comprender la realidad social desde una perspectiva sistémica. Todo esto involucra detectar factores presentes, pero no evidentes en una problemática social, identificar relaciones de causalidad y articular dimensiones sociales, políticas, económicas, etc.” (Ministerio de Educación, 2014, p. 17). Entonces, se espera que, para este nivel de evaluación, los estudiantes puedan identificar causas, dimensiones, soluciones, y efectos sobre un problema histórico o actual.

En tal sentido, para la elaboración del Podcast los estudiantes debían describir las dimensiones sociales, ambientales y geográficas del problema. En otras palabras, era necesario que reconocieran que el conflicto no solo afectaba la estructura ambiental del PNN, sino que incidía en los habitantes de la zona. A la larga, el ejercicio crítico permitió el reconocimiento y análisis de los conflictos políticos y sociales implícitos en el control y manejo de los territorios explorados en clase, tal como se hace evidente en el siguiente ejemplo:

Lo que sucede en el Parque El Cocuy es un problema ambiental, porque los turistas no están cuidando el lugar cuando van, y terminan dañando lo poco que queda después del daño que ya hizo el derretimiento del hielo por el calentamiento global [...] Pero también es un problema social, porque los indígenas 
no quieren más turismo, pero el gobierno sí quiere (Podcast, No. 5).

En este mismo orden de ideas, los estudiantes debieron crear una posible solución que considerara las dimensiones del conflicto, evaluando los posibles impactos sobre los actores; a continuación se presenta un ejemplo de las reflexiones:

Propongo como solución que los habitantes indígenas del PNN El Cocuy hagan una tutela contra las empresas que hacen turismo, y que hagan también un cabildo abierto para limpiar el parque y hacer que nadie más contamine [...], esta solución no afectaría a nadie, ya que, si se hace de forma responsable, todos se verían beneficiados (Podcast, No. 7).

Finalmente, durante el espacio de retroalimentación, los niños compartieron sus estudios a través de un cuaderno virtual colectivo, un escenario creado para que pegaran los enlaces correspondientes a sus trabajos. Cada integrante podría escuchar y evaluar las actividades de sus compañeros utilizando la rúbrica de evaluación. La coevaluación permitió desarrollar la capacidad para apreciar objetivamente, no solo la propia labor, sino la realizada por los compañeros de clase; con ella reconocieron fortalezas, debilidades, alternativas y soluciones. Este ejercicio de conclusión facilitó a los estudiantes involucrarse de forma más activa y auto-dirigida a su proceso de aprendizaje, como parte de una comunidad educativa en formación.

\section{Conclusiones}

El presente artículo tuvo como objetivo exponer una experiencia pedagógica virtual alrededor de la enseñanza de la geografía de Colombia. Se tomó como herramienta el análisis de un problema socioambiental puntual, que resultaría en la creación de un Podcast. En este proyecto cada estudiante debía explicar el problema y sus dimensiones, al tiempo que exponer y contrastar los actores involucrados, junto a sus respectivas perspectivas e intereses, para crear una solución que respondiera a la diversidad de necesidades.

Frente a ello, una primera conclusión se puede referir al uso del Podcast como herramienta didáctica en el aula. Tomando en cuenta los resultados, la elaboración del Podcast facilitó el desarrollo de competencias relativas a las ciencias sociales (pensamiento social, interpretación y análisis de perspectivas, y pensamiento reflexivo y sistémico); al tiempo, el trabajo permitió que los estudiantes pudieran relacionar cuerpos conceptuales concretos (como el páramo, el parque nacional o mecanismos de participación) con problemáticas socioambientales latentes.

Todo el proceso, facilitó el reconocimiento y análisis de las dimensiones implícitas en un conflicto (social, ambiental, política, cultural) y las múltiples percepciones, intereses y necesidades que se trenzan alrededor del uso de la naturaleza. A la larga, el Podcast fue mucho más allá de revisar la apropiación de temas y conceptos, permitió verificar aprendizajes complejos como el análisis de percepciones, el contraste entre intereses y la creación de soluciones integrales y coherentes.

Por otro lado, la estrategia demostró que la Enseñanza para la Comprensión (EPC) es un marco metodológico muy efectivo en la secuenciación del análisis de problemáticas socioambientales para el aprendizaje de la geografía de Colombia. El método 
llevó a utilizar un marco conceptual geográfico de referencia para analizar críticamente un problema socioambiental, al tiempo que a reconocer sus actores, proponer soluciones y evaluar posibilidades.

A su vez, dicho método permitió utilizar fuentes y herramientas diversas para presentar la información de forma creativa, a través de la realización de un Podcast. El paso a paso de la unidad pudo tener un sentido gradual y exponencial, a través del cual cada estudiante tuvo la posibilidad de conocer los objetivos y criterios bajo los cuales debía avanzar en su actividad, desde los que se le estaba evaluando. En últimas, este marco retó al alumno a llevar constantemente sus análisis a niveles más avanzados, permitiéndole utilizar el conocimiento como pilar para solucionar los problemas planteados.

Retomando lo dicho por Souto (1997), Jiménez y Gaite (1996), estas herramientas para el análisis y solución de problemas contemporáneos responden a la formación de ciudadanos del siglo XXI. Reconocer las problemáticas sociales y ambientales como conflictos que envuelven una multiplicidad de percepciones y dimensiones, es un pilar esencial para la consecución de futuros adultos consientes, capaces de utilizar eficiente y efectivamente su conocimiento para solucionar conflictos emergentes.

Finalmente, es importante destacar que el trabajo virtual tuvo una gran relevancia para este proceso didáctico; el acceso a nuevas fuentes, ritmos y contenidos permitió dinamizar un proceso investigativo escolar que generalmente se ve truncado en el ámbito presencial, por las herramientas que no están disponibles. El Podcast y su efectividad como formato digital evidencian la urgente necesidad de explorar las TIC en el aula.
Desde que inició el trabajo desde el ámbito virtual ha sido difícil encontrar estrategias didácticas que generen interés en los estudiantes. Entre otros factores, la situación ha hecho que muchos deban lidiar con una rutina extenuante en casa, sin amigos, juegos o simple contacto con el entorno. A pesar de ello, la producción y grabación del Podcast facilitó la generación de otros espacios de aprendizaje, mediados por las TIC, en donde se contó con la posibilidad de compartir en familia, cambiar la voz para ser otra persona, y buscar música y efectos para acompañar la propia voz. En conclusión, se trató de una actividad emocionante y estimulante, a través de la cual los estudiantes pudieron divertirse mientras aprendían sobre ese entorno geográfico que hoy en día se siente lejano. ․ㅕ 


\section{Referencias}

Cáceres, K. F. (2020). Educación virtual: creando espacios afectivos, de convivencia y aprendizaje en tiempos de COVID-19. CienciAmérica, 9(2), pp. 38-44. DOI: https://doi.org/10.33210/ca.v9i2.284

Cely, A., y Moreno, N. (2008). Cotidianidad y enseñanza geográfica. Bogotá: Códice.

Jiménez, A., y Gaite, M. (1996). Enseñar geografía: de la teoría a la práctica. México: Síntesis.

Martínez, J. (2008). Conflictos ecológicos y justicia ambiental. Papeles, No. 103, pp. 11-27.

Ministerio de Educación Nacional (MEN). (2014). Sistema Nacional de Evaluación Estandarizada de la educación: Alineación del examen saber 11. Bogotá: MEN.

Ministerio de Educación Nacional (MEN). (2010). Lineamientos para la educación virtual en la educación superior. Bogotá: MEN.

Norman, E., y Daza, C. E. (2020). Construcción de contenidos para la enseñanza virtual: retos coyunturales en el confinamiento. Panorama, 14(27), pp. 5-13. DOI: https://doi.org/10.15765/pnrm. v14i27.1517

Rodríguez, A. (2000). Geografía conceptual: enseñanza y aprendizaje de la geografía en la educación básica primaria. Bogotá: Tercer Mundo.
Souto, Xosé. (1997) ¿Cómo abordar problemas ambientales y sociales desde el aula? Valencia: Nau Libres.

Stone, M. S. (1999). Enseñanza para la comprensión. Buenos Aires: Paidós. 